\title{
Power-controlled phase-matching and instability of CW propagation in multicore optical fibers with a central core
}

\author{
A. M. Rubenchik, ${ }^{1}$ E. V. Tkachenko, ${ }^{2}$ M. P. Fedoruk, ${ }^{2,3}$ and S. K. Turitsyn ${ }^{3,4, *}$ \\ ${ }^{1}$ Lawrence Livermore National Laboratory, Livermore, California 94550, USA \\ ${ }^{2}$ Institute for Computational Technologies, SB RAS, Novosibirsk 630090, Russia \\ ${ }^{3}$ Novosibirsk State University, Novosibirsk 630090, Russia \\ ${ }^{4}$ Aston Institute of Photonic Technologies, Aston University, Birmingham B4 7ET, UK \\ *Corresponding author: s.k.turitsyn@aston.ac.uk
}

Received August 5, 2013; accepted August 30, 2013;

posted September 16, 2013 (Doc. ID 195226); published October 14, 2013

\begin{abstract}
We present modulation instability analysis including azimuthal perturbations of steady-state continuous wave (CW) propagation in multicore-fiber configurations with a central core. In systems with a central core, a steady CW evolution regime requires power-controlled phase matching, which offers interesting spatial-division applications. Our results have general applicability and are relevant to a range of physical and engineering systems, including high-power fiber lasers, optical transmission in multicore fiber, and systems of coupled nonlinear waveguides. (c) 2013 Optical Society of America

OCIS codes: (190.4360) Nonlinear optics, devices; (060.4370) Nonlinear optics, fibers.

http://dx.doi.org/10.1364/OL.38.004232
\end{abstract}

The fast developing technology of optical multicore fiber (MCF) offers the possibility of spatial-division multiplexing (SDM), enabling a scale-up in transmission capacity per fiber that is a crucial challenge in optical communications [1,2]. It is often assumed that, though the addition of spatial channels is technically different from appending spectral channels, in terms of general consideration and system management, SDM in many senses is quite similar to the technology of wave-division multiplexing (WDM). One of the goals of this work is to point out that in MCF spatial channels may be nonequal, and the transfer of channel management approaches from WDM to SDM is not always straightforward. For instance, in WDM the signal-to-noise ratio (SNR) is often defined per spectral channel, assuming close to uniform spectral-power density distribution between channels. As we will show below, in MCF configurations with a central core (see Fig. 1, e.g., seven-core MCF [1, 2]), a steady-state CW propagation with equal power in all spatial channels is not possible at all, making in this system the regime with equal spatial-channel powers inherently more prone to cross talks. Therefore, the SNR in SDM systems should be defined in a way different to standard WDM systems, taking into account difference between spatial channels.

Another important emerging application of MCF is in the field of high-power fiber lasers [3]. Nonlinear effects limit the power that can be transmitted in a single-mode fiber. In a MCF, light in each core may be transmitted below threshold of the detrimental nonlinear effects while the total coherently combined power can be high. MCF technology is used in high-brightness sources based on the coherent combining technique.

In both major applications of MCF, nonlinear interactions between light in different cores can critically affect system performance. Therefore, knowledge of the limits imposed by the nonlinearity on coherent transmission of light through the MCF is of high practical importance. Despite this, the nonlinear dynamics of light in MCF is not yet well studied.

The mathematical analysis of nonlinear wave propagation in MCFs is also a generic problem with numerous links to the theory of nonlinear discrete systems (see e.g., [4-19] and references therein). As was already demonstrated in [4] in MCF with nonequal cores (the most simple and general case is $N$ peripheral cores surrounding the central core), phase matching and stable coherent propagation are possible only due to nonlinear effects for certain power balance between cores. In [4] the stability problem of steady-state propagation was solved in the radial approximation without consideration of azimuthal perturbations. It has been shown in [ $\underline{4}]$ that, surprisingly, even at high light intensities, stable coherent propagation is possible. In this Letter, we extend the stability analysis to the important case of azimuthal perturbations, and we account for the possibility of power transfer between peripheral cores (see Fig. 1). In the optical communication context, our results provide the underlying theory explaining why in the spatial-division-multiplexing technique with MCF having a central core, spatial power distribution (power per spatial channel) should not be uniform, but instead has to be adjusted to the MCF geometry, as described below.

The basic model considered here is a version of the discrete nonlinear Schrödinger equation:

$$
i \frac{\partial A_{k}}{\partial z}+\sum_{m=0}^{N} C_{k m} A_{m}+2 \gamma_{k}\left|A_{k}\right|^{2} A_{k}=0 ; \quad k=0, \ldots, N
$$

Here $A_{k}$ is the field in the $k$-th core $(k=1,2,3, \ldots, N)$, with $A_{0}$ being the field in the central core, and $C_{m k}$ is the coupling coefficient between modes $m$ and $k$; $C_{k, k \pm 1}=C_{1}(k \neq 0), C_{k, 0}=C_{0}, C_{k k}=\beta_{k}$. The coefficients related to wave numbers and nonlinearity in peripheral 
cores, without loss of generality, are assumed to be equal $\left(\beta_{k}=\beta_{1}\right.$ and $\left.\gamma_{k}=\gamma_{1}\right)$. We assume, though, that in general nonlinear coefficients in central and peripheral cores can be different. It is convenient to rewrite these equations in normalized units:

$$
i \frac{\partial U_{0}}{\partial z}+\frac{1}{N} \sum_{k=1}^{N} U_{k}+\frac{2 N \gamma_{0}}{\gamma_{1}}\left|U_{0}\right|^{2} U_{0}=0
$$

$i \frac{\partial U_{k}}{\partial z}+(\kappa-2 C) U_{k}+C\left(U_{k+1}+U_{k-1}\right)+U_{0}+2\left|U_{k}\right|^{2} U_{k}=0$.

Here we introduced normalized variables: $A_{0, k}=$ $\sqrt{P_{0,1}} U_{0, k} e^{i \beta_{0} L z} ; \quad z^{\prime}=z / L ; \quad L=1 /\left(C_{0} \sqrt{N}\right), \quad C=C_{1} /$ $\left(C_{0} \sqrt{N}\right)$, and $P_{0}=N P_{1}=N^{3 / 2} C_{0} / \gamma_{1}, \kappa=\left(\beta_{1}-\beta_{0}+2 C_{1}\right) /$ $\left(C_{0} \sqrt{N}\right.$ ). The total (normalized by $P_{0}$ ) power $P_{t}=$ $N\left(\left|U_{0}\right|^{2}+\left|U_{1}\right|^{2}\right)$ is a conserved quantity.

As was pointed out in [4], in the case of multiple peripheral cores surrounding a central core, even the existence of a steady-state solution is nontrivial. To provide for coherent steady-state $\mathrm{CW}$ light evolution in multiple cores, the difference in propagation constants has to be compensated by the nonlinear phase shifts:

$$
\begin{gathered}
\left\{U_{0}, U_{k}\right\}=\{A, B\} \times e^{i \lambda z}, \quad \Gamma=\frac{B}{A}, \\
|A|^{2}=\frac{P_{t}}{N\left(1+\Gamma^{2}\right)}, \quad \lambda=\Gamma+\frac{2 \gamma_{0} P_{t}}{\gamma_{1}\left(1+\Gamma^{2}\right)}, \\
\Gamma^{4}-\left(\kappa+\frac{2 P_{t}}{N}\right) \Gamma^{3}-\left(\kappa-\frac{2 \gamma_{0} P_{t}}{\gamma_{1}}\right) \Gamma-1=0 .
\end{gathered}
$$

In this Letter, we limit analysis only by the case of inand out-of-phase fields $A$ and $B$, meaning real values of $\Gamma$. More general case will be presented elsewhere. The steady-state solutions in such a system are possible only with a certain imbalance (given by the factor) between powers propagating $\Gamma^{2}=B^{2} / A^{2}$ in central $(A)$ and ring $(B)$ cores. The physics of this effect is rather transparent-the power split is due to the nonlinear phase-shift contribution to the phase-matching condition required for coherent propagation in multiple cores. The amount of power that has to be coupled to each core for steadystate evolution given by the solution of Eq. (6) depends on four parameters: (1) $N$; (2) input power $P_{\text {in }}$ (or total power $P_{t}$ ); (3) the linear phase mismatch $\kappa$; and (4) the ratio between the nonlinear coefficients $\gamma_{0} / \gamma_{1}$ (see Fig. 2). To get the idea of the solution structure, consider the practically important case $P_{t} \gg 1$. In this case, from Eq. (6) we will get explicitly four families of solutions. In $\Gamma_{1}=2 P_{t} / N$ and $\Gamma_{3}=\gamma_{1} /\left(2 \gamma_{0} P_{t}\right)$, most of the energy propagates are in the ring or central core, respectively. For $\Gamma_{2,4}= \pm \sqrt{\gamma_{1} N / \gamma_{0}}$, the ratio of energy in the ring and the central core is independent of the propagating power. Negative $\Gamma$ means out-of phase fields in the central and peripheral cores.

Consider now the stability of steady-state solutions of Eqs. (4)-(6), the analogue of the MI for a low-dimension discrete system. The small amplitude disturbance is taken in a standard form $\left\{U_{0}, U_{k}\right\}=\{A+a+i b, B+$ $\left.c+i d+(f+i h) e^{i s k}\right\} \times e^{i \lambda z}$ and $k=1,2,3, \ldots, N$. It is easy to see that perturbations of $U_{k}$ proportional to $\exp [p z]$ have an isotropic ( $k$ independent) part (considered in [4]) and an angular ( $k$ dependent) contribution. Straightforward analysis shows that the angular part of perturbations is independent of the isotropic part, and the increment (growth rate) of instability due to angular perturbations is

$$
p_{l}^{2}=\left(\frac{1}{\Gamma_{l}}+4 C \sin ^{2}\left[\frac{s}{2}\right]\right)\left(\frac{4 \Gamma_{l}^{2} P_{t}}{N\left(1+\Gamma_{l}^{2}\right)}-\frac{1}{\Gamma_{l}}-4 C \sin ^{2}\left[\frac{s}{2}\right]\right),
$$

here $l=1,2,3,4$ corresponds to four branches of CW steady states. Recall that $C=C_{1} /\left(C_{0} \sqrt{N}\right)$.

From the periodicity condition with $m=1,2,3, \ldots, N$, note that the expression for the increment in Eq. (7) looks structurally exactly as the classical formula for MI growth rate [20,21]: $p_{l}^{2}=\Lambda\left(\alpha P_{t}-\Lambda\right)$. However, here $\Lambda=1 / \Gamma_{l}+4 C \sin ^{2}[s / 2]$ is a discrete variable. Equation (7) without the term $1 / \Gamma_{l}$ describes the modulation instability (MI) in discrete systems (see e.g., $[7,8,12])$ and in the limit $s \ll 1$ transforms into conventional MI growth rate $[\underline{20}, 21]$. We see that the effect of a central fiber $1 / \Gamma$ plays a stabilizing role for positive $\Gamma$. For negative $\Gamma$, mode $\Gamma_{4}$, the presence of the central core enhances the instability. We discuss below only the new features introduced by the possibility of a positive $\Gamma$.

The minimal value of $s=2 \pi / N, m=1$ gives the threshold (in power) of the azimuthal MI:

$$
\frac{4 \Gamma_{l}^{2} P_{t}}{N\left(1+\Gamma_{l}^{2}\right)}<\frac{1}{\Gamma_{l}}+4 C \sin ^{2}\left[\frac{\pi}{N}\right] .
$$

Here $\Gamma_{l}=\Gamma_{l}\left(N, P_{t}\right)$ is a function of $N$ and $P_{t}$, making this equation an implicit condition on power. For continuous case $N \rightarrow \infty$, MI has no threshold. However, both the discreteness and presence of a central core (with positive $\Gamma$ ) suppress the instability. The maximum growth rate $p_{\max }$ is reached at

$$
\frac{2 \Gamma_{l}^{2} P_{t}}{N\left(1+\Gamma_{l}^{2}\right)}=\frac{1}{\Gamma_{l}}+4 C \sin ^{2}[\pi m / N],
$$

and $p_{\max }=2 \Gamma_{l}^{2} P_{t} /\left[N\left(1+\Gamma_{l}^{2}\right]\right.$. The instability is developed at the length $L \sim 1 / p_{\max }$.

Analysis of the analytical asymptotic solutions (valid at $P_{t} \gg 1$ ) shows that only $\Gamma_{3}$ is a stable solution (in some range of parameters in the plane $\left(N, P_{t}\right)$ shown by color in Fig. 3). All other solutions $\Gamma_{1,2,3}$ are unstable. In the case of angular perturbations, it is not possible to derive an exact analytical expression defining the stability zones for all values of $P_{t}$. The stability boundaries for $P_{t}$ not large can be found numerically. The calculations for the sufficient conditions of stability and instability for 

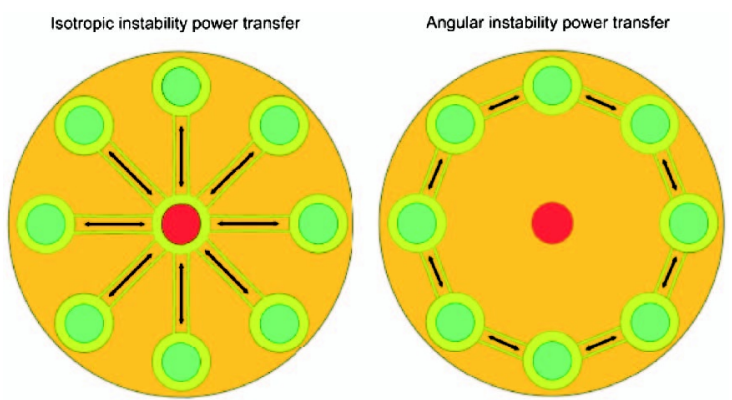

Fig. 1. Schematic depiction of the MCF and power exchanges between cores.

$\Gamma_{3}\left(N, P_{t}\right)$ and, thus, define stable and unstable areas in the plane $\left(N, P_{t}\right)$ as illustrated by Fig. 3 . The threshold curve in Eq. (8) for $\Gamma_{3}$, when a big fraction of energy is concentrated in the central core, is presented in Fig. 3. The increment of instability $p^{2}$ as a function of $s$ for $\bar{\Gamma}_{2}$ and $\Gamma_{3}$ is depicted in Fig. 4 for various $N$ and $P_{t}$. We see that for the fixed $P_{t}$, the growth rate is decreased with the number of cores. The intensity in every core is going down with $N$ increase, and the $\mathrm{MI}$ is determined by the local radiation intensity. However, one can see that the growth decrease is slower then $1 / N$, which indicates that the increase in core numbers makes MCF more susceptive to the MI.

The maximum power $P_{\max }$ that can be transported in a single core in unrelated to the threshold power $P_{\text {th }}$ for the instability calculated above. Hence, our analysis paves the way for design of MCF that will support the stable propagation of the total power $P_{t}$ well above $P_{\max }$. The coherent output from the fiber end can be then combined in one beam leading to the compact and efficient beam-combining scheme.

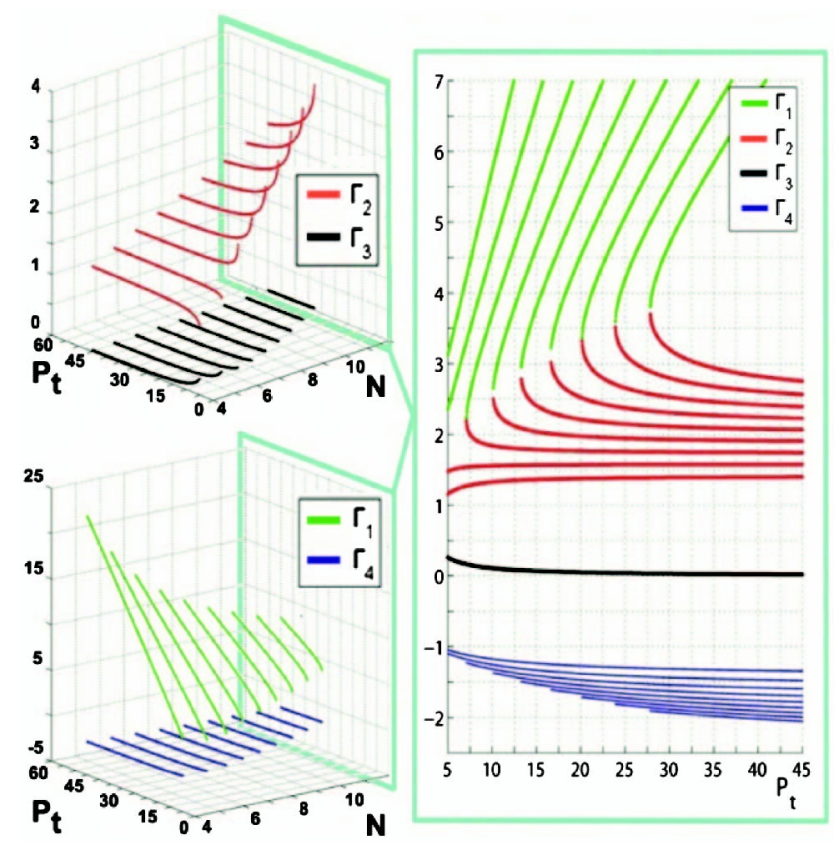

Fig. 2. Four values of $\Gamma$ corresponding to different power splits between cores as functions of total input power; here $\gamma_{0} / \gamma_{1}=0.5$ and $\kappa=1$. Here different curves for each branch correspond to $N$ varying from 3 to 12 (from the bottom to the top, from left to right for green curve).

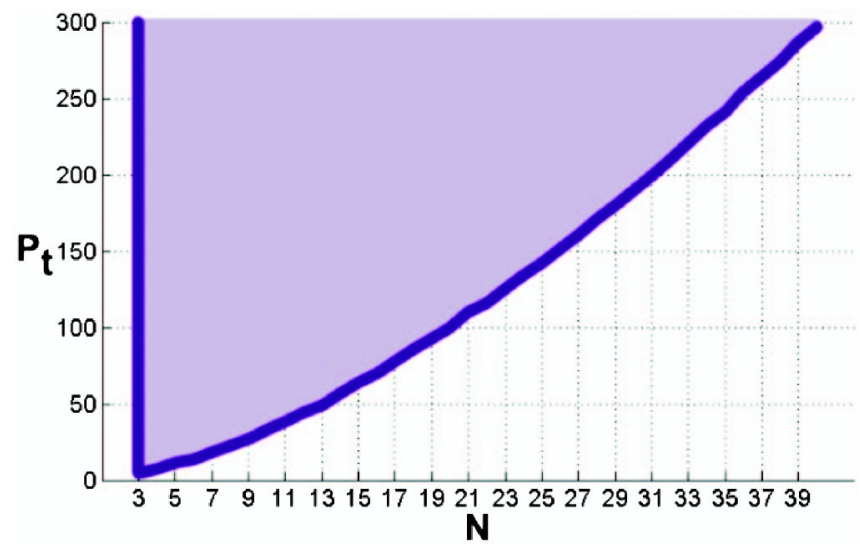

Fig. 3. Angular stability area (shown by color) of $\Gamma_{3}\left(\Gamma_{1}, \Gamma_{2}\right.$, and $\Gamma_{4}$ are unstable) corresponding to different values of the total power $P_{t}$ and $N$; here $\gamma_{0} / \gamma_{1}=1$ and $\kappa=1$.

Nonlinear instability leads to periodic exchange of power between cores. With a growing number of cores, these oscillations may become stochastic. As a result, the instability makes power dynamics uncontrollable. Therefore knowledge of the criteria of instability onsets is important for the design of physical systems and devices based on MCF. The considered instability is an extreme discrete limit of the conventional MI in the continuous media and discrete systems (see e.g., [주요, 12-16,20-22] and references therein). Due to the generality of the master equations, we anticipate that our results may provide a new outlook at the traditional arrays of coupled nonlinear waveguides and nonlinear lattice research [5-19]. We would like to stress also that, although the presented analysis deals with the CW propagation, the obtained results are applied to time-dependent fibercommunication channels. In that case, the power should be understood as time-averaged signal characteristics, such as the average signal power. Note also that the presented theory can be generalized in a straightforward manner to pulse propagation in MCF. The efficiency of nonlinear matching through a fundamental soliton coupling from one fiber into another studied in recent work

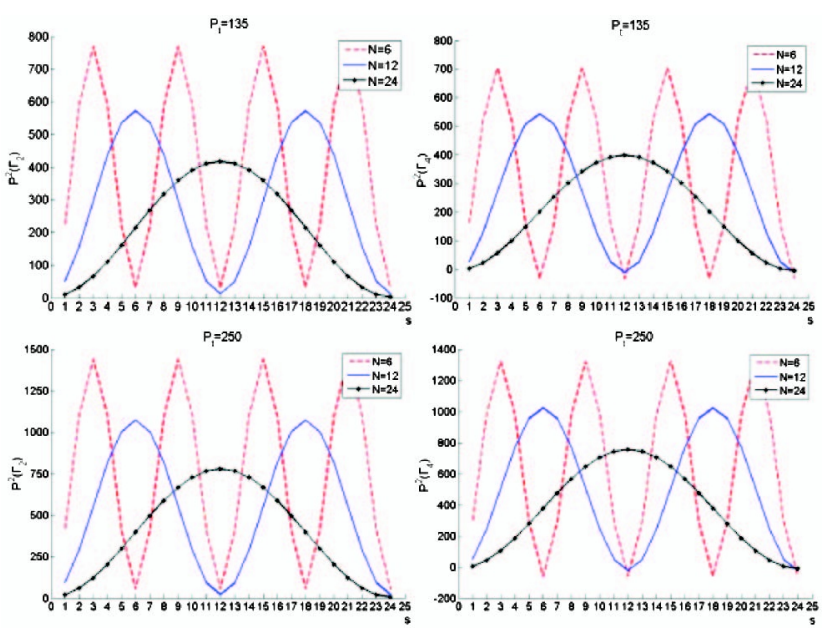

Fig. 4. Increment of instability $p^{2}$ as a function of $s$ for $\Gamma_{2}$ and $\Gamma_{4}$ corresponding to different $P_{t}$ and $N$ (red dashed line: $N=6$; solid blue: $N=12$; solid-dotted black: $N=24$ ). 
[23] has similarities with CW power matching examined here. This paves the way to numerous applications; for instance, a controlled Raman red-shift and supercontinuum generation.

To conclude, in this Letter we have presented a theory of an instability, including azimuthal perturbations of a steady-state CW propagation in multicore-fiber configurations with a central core. In MCF with a central core, a steady-state CW propagation requires power-supported phase matching. This has an important consequence for SDM. In the context of optical-fiber communications, using MCF our results can be understood in the following way: in the considered system of MCFs with a central core, a power per spatial channel cannot be uniform in a stable propagation. Stable propagation requires certain disbalance between the power in a central core and other given by $\Gamma_{l}^{2}$. This disbalance depends on MCF geometry and other system parameters as described above. In particular, this means that the SNR should not be introduced per spatial channel and more sophisticated definitions are required. The developed theory is rather generic and has a number of applications from high-power fiber lasers to bulk nonlinear waveguiding systems.

This work was performed under the auspices of the U.S. Department of Energy by Lawrence Livermore National Laboratory under Contract DE-AC52-07NA27344. The financial support of the EPSRC program grant UNLOC (EP/J017582/1), grant of the Ministry of Education and Science of the Russian Federation (agreement no. 14.B25.31.0003), the European Research Council (ULTRALASER) and the Marie Curie IRSES program is acknowledged.

\section{References}

1. F. Y. M. Chan, A. P. T. Lau, and H.-Ya. Tam, Opt. Express 20 , 4548 (2012)

2. B. Zhu, T. F. Taunay, M. F. Yan, J. M. Fini, M. Fishteyn, E. M. Monberg, and F. V. Dimarcello, Opt. Express 18, 11117 (2010).
3. B. M. Shalaby, V. Kermene, D. Pagnoux, A. DesfargesBerthelemot, A. Barthelemy, A. Popp, M. Abdou Ahmed, A. Voss, and T. Graf, Appl. Phys. B 100, 859 (2010).

4. S. K. Turitsyn, A. M. Rubenchik, M. P. Fedoruk, and E. Tkachenko, Phys. Rev. A 86, 031804(R) (2012).

5. D. N. Christodoulides and R. I. Joseph, Opt. Lett. 13, 794 (1988).

6. C. Schmidt-Hattenberger, U. Truschel, and F. Lederer, Opt. Lett. 16, 294 (1991).

7. Yu. S. Kivshar and M. Peyard, Phys. Rev. A 46, 3198 (1992).

8. A. B. Aceves, C. De Angelis, G. G. Luther, and A. M. Rubenchik, Opt. Lett. 19, 1186 (1994).

9. A. B. Aceves, G. Luther, C. De Angelis, A. Rubenchik, and S. K. Turitsyn, Phys. Rev. Lett. 75, 73 (1995).

10. R. Morandotti, H. Eisenberg, U. Peschel, Y. Silberberg, and J. S. Aitchison, Phys. Rev. Lett. 83, 2726 (1999).

11. P. G. Kevrekidis, K. O. Rasmussen, and A. R. Bishop, Int. J. Mod. Phys. B 15, 2833 (2001).

12. L. Hadžievski, M. Stepić, and M. M. Škorich, Phys. Rev. B 68, 014305 (2003).

13. A. A. Maier, Phys. Usp. 38, 991 (1995).

14. A. A. Maier, Phys. Usp. 39, 1109 (1996).

15. K. Hizaidis, S. Droulias, I. Tsopelas, N. K. Efremidis, and D. N. Christodoulides, Int. J. Bifurcation Chaos 16, 1739 (2006).

16. J. Fleischer, G. Bartal, O. Cohen, T. Schwartz, O. Manela, B. Freedman, M. Segev, H. Buljan, and N. Efremidis, Opt. Express 13, 1780 (2005).

17. E. W. Laedke, K. H. Spatschek, V. K. Mezentsev, S. L. Musher, I. V. Ryzhenkova, and S. K. Turitsyn, J. Exp. Theor. Phys. Lett. 62, 677 (1995).

18. E. W. Laedke, K.-H. Spatschek, S. K. Turitsyn, and V. K. Mezentsev, Phys. Rev. E 52, 5549 (1995).

19. E. W. Laedke, K. H. Spatschek, and S. K. Turitsyn, Phys. Rev. Lett. 73, 1055 (1994).

20. F. Abdullaev, S. Darmanyan, and J. Garnier, in Progress in Optics, E. Wolf, ed. (Elsevier, 2002), 44, p. 303.

21. V. E. Zakharov and L. A. Ostrovsky, Physica D 238, 540 (2009).

22. C. Huang, Y. Lai, S. Cheng, and W. Hsieh, Opt. Express 17, 1299 (2009).

23. C. Agger, S. T. Srensen, C. L. Thomsen, S. R. Keiding, and O. Bang, Opt. Lett. 36, 2596 (2011). 